\title{
The loss of ions from Venus through the plasma wake
}

\author{
S. Barabash ${ }^{1}$, A. Fedorov ${ }^{2}$, J. J. Sauvaud ${ }^{2}$, R. Lundin ${ }^{1}$, C. T. Russell ${ }^{3}$, Y. Futaana ${ }^{1}$, T. L. Zhang ${ }^{4}$, H. Andersson ${ }^{1}$, \\ K. Brinkfeldt ${ }^{1}$, A. Grigoriev ${ }^{1}$, M. Holmström ${ }^{1}$, M. Yamauchi ${ }^{1}$, K. Asamura ${ }^{5}$, W. Baumjohann ${ }^{4}$, H. Lammer ${ }^{4}$, \\ A. J. Coates $^{6}$, D. O. Kataria ${ }^{6}$, D. R. Linder ${ }^{6}$, C. C. Curtis ${ }^{7}$, K. C. Hsieh ${ }^{7}$, B. R. Sandel ${ }^{7}$, M. Grande ${ }^{8}$, H. Gunell ${ }^{9}$, \\ H. E. J. Koskinen ${ }^{10,11}$, E. Kallio ${ }^{11}$, P. Riihelä ${ }^{11}$, T. Säles ${ }^{11}$, W. Schmidt ${ }^{11}$, J. Kozyra ${ }^{12}$, N. Krupp ${ }^{13}$, M. Fränz ${ }^{13}$, J. Woch $^{13}$, \\ J. Luhmann ${ }^{14}$, S. McKenna-Lawlor ${ }^{15}$, C. Mazelle ${ }^{2}$, J.-J. Thocaven ${ }^{2}$, S. Orsini ${ }^{16}$, R. Cerulli-Irelli ${ }^{16}$, M. Mura ${ }^{16}$, \\ M. Milillo ${ }^{16}$, M. Maggi ${ }^{16}$, E. Roelof ${ }^{17}$, P. Brandt ${ }^{17}$, K. Szego $^{18}$, J. D. Winningham ${ }^{19}$, R. A. Frahm ${ }^{19}$, J. Scherrer ${ }^{19}$, \\ J. R. Sharber ${ }^{19}$, P. Wurz ${ }^{20} \&$ P. Bochsler ${ }^{20}$
}

Venus, unlike Earth, is an extremely dry planet although both began with similar masses, distances from the Sun, and presumably water inventories. The high deuterium-to-hydrogen ratio in the venusian atmosphere relative to Earth's also indicates that the atmosphere has undergone significantly different evolution over the age of the Solar System ${ }^{1}$. Present-day thermal escape is low for all atmospheric species. However, hydrogen can escape by means of collisions with hot atoms from ionospheric photochemistry ${ }^{2}$, and although the bulk of $\mathrm{O}$ and $\mathrm{O}_{2}$ are gravitationally bound, heavy ions have been observed to escape ${ }^{3}$ through interaction with the solar wind. Nevertheless, their relative rates of escape, spatial distribution, and composition could not be determined from these previous measurements. Here we report Venus Express measurements showing that the dominant escaping ions are $\mathrm{O}^{+}, \mathrm{He}^{+}$and $\mathrm{H}^{+}$. The escaping ions leave Venus through the plasma sheet (a central portion of the plasma wake) and in a boundary layer of the induced magnetosphere. The escape rate ratios are $Q\left(\mathrm{H}^{+}\right) / Q\left(\mathrm{O}^{+}\right)=1.9 ; Q\left(\mathrm{He}^{+}\right) / Q\left(\mathrm{O}^{+}\right)=0.07$. The first of these implies that the escape of $\mathrm{H}^{+}$and $\mathrm{O}^{+}$, together with the estimated escape of neutral hydrogen and oxygen, currently takes place near the stoichometric ratio corresponding to water.

Our knowledge of the interaction of Venus with the solar wind and its role in the acceleration of ions is based mainly on the long-lived Pioneer Venus orbiter mission, which carried a limited plasma analyser with low time resolution ${ }^{4}$ and an ion-neutral mass spectrometer with a restricted energy range of up to $\sim 100 \mathrm{eV}$ (ref. 5). These observations identified $\mathrm{O}^{+}$ions only indirectly, through their energy spectra. However, they showed that the distribution of escaping oxygen ions is controlled by the solar-wind convection electric field, a distinctive signature of the ion pick-up process ${ }^{6}$. The comprehensive plasma package on Venus Express comprises an ion mass analyser, an electron spectrometer, and energetic neutral atom imagers ${ }^{7}$. The energy range of the charged particle sensors, from $1 \mathrm{eV}$ (measurements below $10 \mathrm{eV}$ may be affected by the spacecraft potential) to $15 \mathrm{keV}$ (electrons) and to $36 \mathrm{keV}$ (ions), is well suited for measurements of plasma escape. The magnetic field data are provided by the Venus Express magnetometer ${ }^{8}$. The polar, highly elliptical orbit, with a pericentre of $250-350 \mathrm{~km}$ and an apocentre of $66,000 \mathrm{~km}$, is optimal for studying the escaping planetary ions because it covers the region of the interaction of the solar wind near the terminator and the region directly downstream of the planet.

During pericentre flybys the spacecraft crosses the bow shock, where the supersonic solar-wind plasma is heated and deflected, and enters the magnetosheath, a region of heated subsonic solarwind flow (Fig. 1a). The magnetic field properties of these regions are treated in another paper in this issue $e^{9}$. Then the spacecraft penetrates the induced magnetosphere boundary, where the solar-wind flux drops and the planetary ions $\mathrm{O}^{+}, \mathrm{He}^{+}$and $\mathrm{H}^{+}$appear (Fig. 1e-g). These three ion species are reliably identified on all orbits, predominantly in the plasma sheet (Fig. 1b), a region separating the two lobes of the induced magnetotail possessing opposite magnetic field directions. In the magnetic field data the plasma sheet is readily identified by the change in the sign of the magnetic field component along the Venus-Sun line $\left(B_{x}\right)$ and a decreased field magnitude (Fig. 1c). A plasma sheet should contain plasma whose thermal pressure balances the magnetic pressure from the surrounding lobes. In particle data it is most readily identified by the increase in the electron fluxes (Fig. 1d). The plane of the plasma sheet is aligned with the electric field formed from solar-wind convection around the planet (Fig. 1b). The $\mathrm{O}^{+}, \mathrm{He}^{+}$and $\mathrm{H}^{+}$ions of planetary origin are also found close to the induced magnetosphere boundary but at a much lower energy (around 7:20 and 8:10 UT in Fig. 1).

Whereas the $\mathrm{O}^{+}$ions can only be of planetary origin, $\mathrm{He}^{+}$ions may originally have been solar-wind $\alpha$-particles having undergone recombination, and the $\mathrm{H}^{+}$ions could also be solar-wind protons. However, the identical shapes of their energy spectra and similar spatial distributions confirm the planetary origin of these ion species. Inside the plasma sheet the ions have different energies, in accordance with their masses (Fig. 2). In the $+Z_{\text {Vse }}$ hemisphere (Vse being the Venus-solar-electrical coordinate system), $\sim 0.5-1.0-\mathrm{keV} \mathrm{O}^{+}$ions are separated from the $100-200-\mathrm{eV} \mathrm{He}^{+}$ions, whereas the $\mathrm{H}^{+}$ions with energies below $100 \mathrm{eV}$ fill both sides $\left(+Y_{\mathrm{Vse}}\right.$ and $\left.-Y_{\mathrm{Vse}}\right)$ of the plasma sheet (Fig. 2b, c). At the induced magnetosphere boundary, all three components have an energy below $50 \mathrm{eV}$ (Fig. 2d).

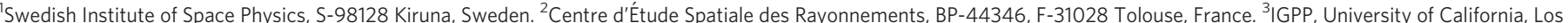

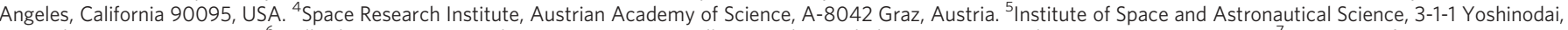

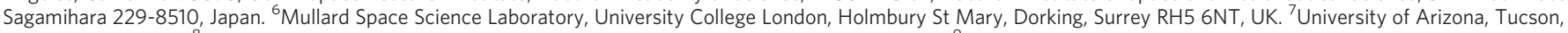

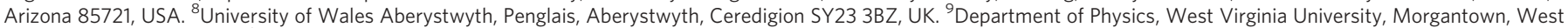

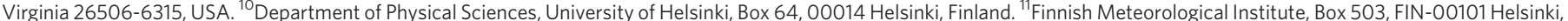

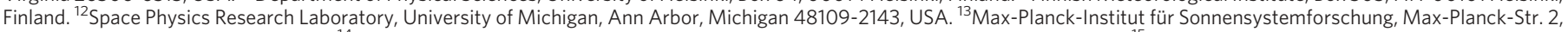

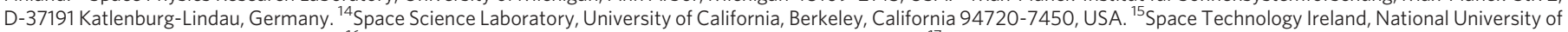

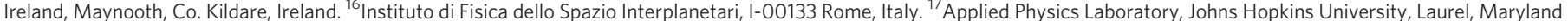

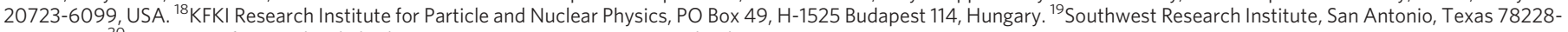
0510, USA. ${ }^{20}$ University of Bern, Physikalisches Institut, CH-3012 Bern, Switzerland. 

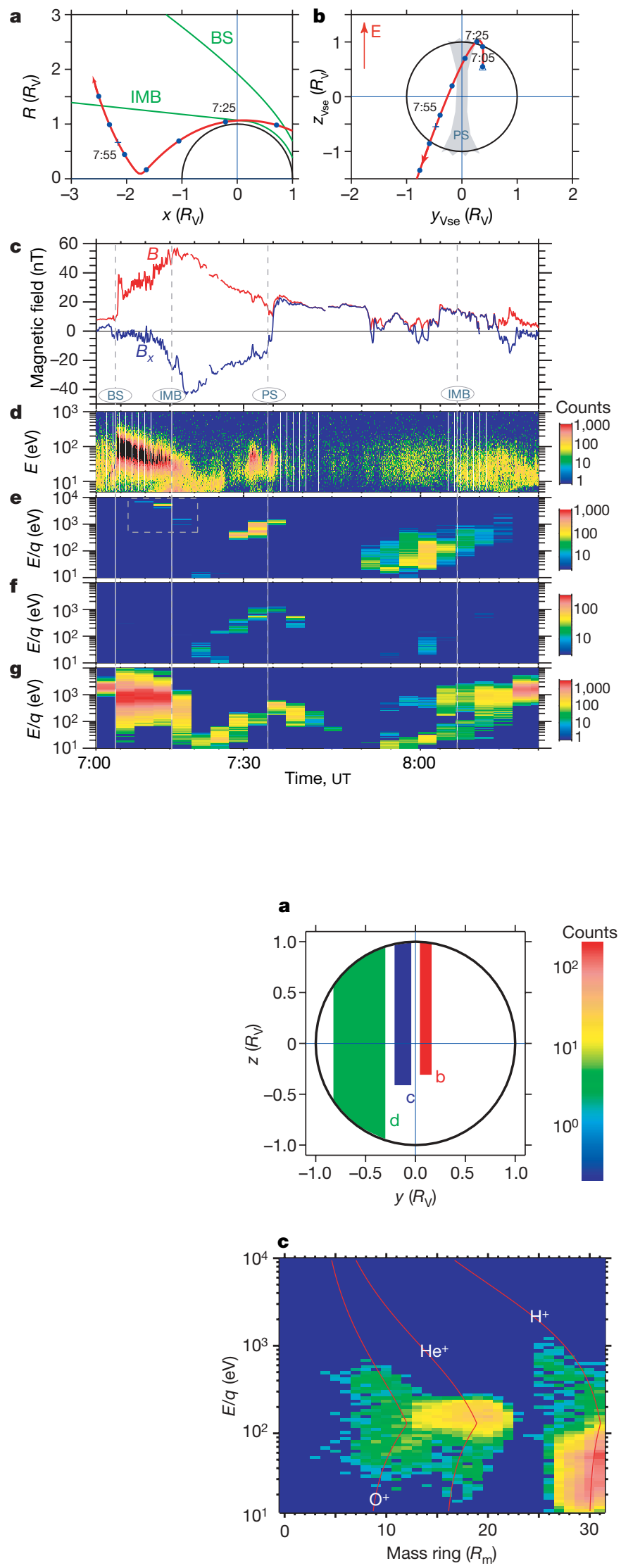

Figure 2 Composition and energy distribution of the escaping plasma in three spatial regions. $a$, The regions labelled by $c$ and $d$ where energy-mass matrixes were collected in the Vse coordinate system (see legend to Supplementary Fig. 2). b-d, The energy-mass matrixes accumulated over 33 orbits during the period from 2006 May 18 to 2006 December 30 and collected in regions b-d in panel a, showing a plasma sheet matrix for the $+Y$
Figure 1 | Venus Express orbit, and plasma and magnetic field measurements on 2006 December 12. a, The Venus Express orbit in cylindrical coordinates, with $x$ pointing towards the Sun, and $R$ being the distance to the $x$ axis (in Venus radii, $R_{\mathrm{V}}=6,051 \mathrm{~km}$ ). The green lines are the averaged $^{22}$ positions of the bow shock (BS) and induced magnetosphere boundary (IMB ${ }^{23}$, and the red line is the Venus Express orbit. $\mathbf{b}$, The Venus Express orbit in the Venus-solar-electrical coordinate system (Vse). In this system $x$ points to the Sun, and $z$ is in the plane containing the convection electric field $E=-V_{\mathrm{sw}} \times B$ (where $V_{\mathrm{sw}}$ is the velocity of the solar wind, $B$ is the interplanetary magnetic field and $\times$ denotes the vector cross product); $y$ completes the orthogonal coordinate system. The plasma sheet (PS) is identified by the grey area. The direction of the convection electric field is labelled E. c, The magnetic field magnitude (red line) and $B_{x}$ component (blue line). $\mathbf{d}-\mathrm{g}$, Energy-time spectrograms for electrons (d), $\mathrm{O}^{+}$ions (e), $\mathrm{He}^{+}$ions (f) and protons (g) (see legend to Supplementary Fig. 1). Venus Express crosses the IMB at 7:02 UT, passes the PS at 7:34 UT and enters the magnetosheath again at 8:08 UT. The pick-up $\mathrm{O}^{+}$ions in the magnetosheath at about 7:13 UT are bounded by a dashed rectangle. $E / q$, energy/charge.

At Venus the ion acceleration occurs by three mechanisms: by ion pick-up (that is, by acceleration in the convection electric field), by instabilities at the induced magnetosphere boundary resulting in detached plasma clouds ${ }^{10}$, and by polarization electric fields at low altitudes in the night-side ionosphere ${ }^{11}$ where the field is nearly radial.

We observed two different populations of the accelerated planetary ions, namely ions escaping through the plasma sheet and ions escaping through the induced magnetosphere boundary layer. The energy distributions of these two populations are different. The ratio of the $\mathrm{O}^{+} / \mathrm{He}^{+} / \mathrm{H}^{+}$energies in the plasma sheet is about 4:2:1 (Fig. 2b, c). The ion energy is therefore a function of mass. That points to ion pick-up as an acceleration mechanism in the plasma sheet, in general agreement with the previous simulations for $\mathrm{O}^{+}$(ref. 12). However, ordinary pick-up would result in a 16:4:1 ratio of the $\mathrm{O}^{+} / \mathrm{He}^{+} / \mathrm{H}^{+}$
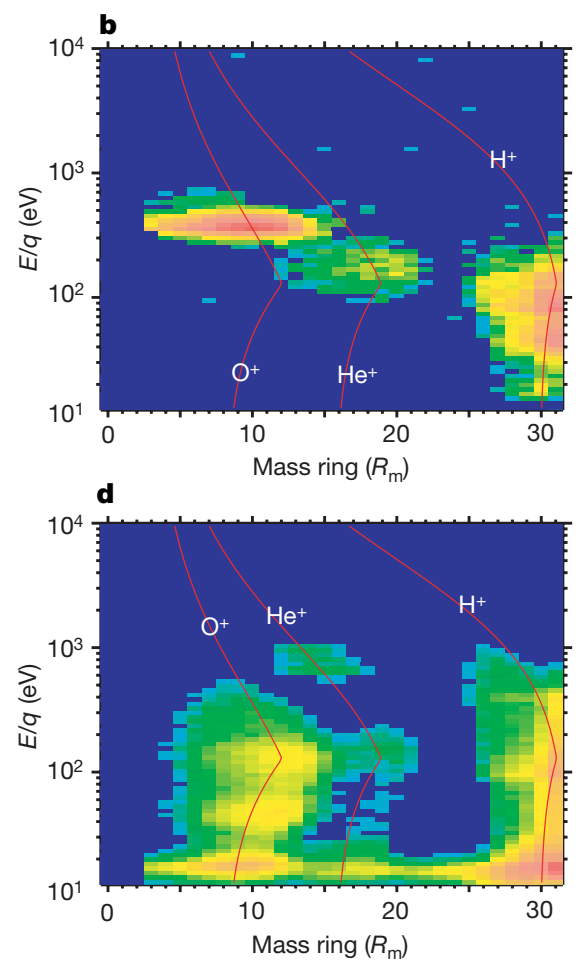

lobe (b), a plasma sheet matrix for the $-Y$ lobe (c), and a matrix for the IMB layer $(\mathbf{d})$. Vertical axes are energy/charge $(E / q)$, the horizontal axes are the position of the ion impact on the detector (a sensor mass ring, $R_{\mathrm{m}}$ ), and the colour codes the counts that were accumulated over all directions and averaged over occurrences. The red lines represent constant mass. 
energies. The difference may be due to species-dependent assimilation into the plasma flow. The ion pick-up process also operates, for example, in the magnetosheath region, in general agreement with the previous simulations for $\mathrm{O}^{+}$(ref. 12). See a clear example of 7-8-keV $\mathrm{O}^{+}$ions at 7:15 UT in Fig. $1 \mathrm{e}$ and $700-800-\mathrm{eV} \mathrm{He}{ }^{+}$ions in Fig. $2 \mathrm{~d}$. The acceleration of the low-energy ions $(<50 \mathrm{eV})$ detected at the induced magnetosphere boundary (Fig. $2 \mathrm{~d}$ ) is possibly due to a polarization electric field ${ }^{11}$ resulting from the magnetic pressure gradient and the gravitational separation of ions and electrons along draped, stretched magnetic field lines. Because of the low plasma velocity in the boundary layer close to the induced magnetosphere boundary, the convection electric field is low and the accelerated ionospheric ions move away from the planet along magnetic field lines in a manner similar to that of the polar wind on Earth.

It is an open question whether the low-energy $(<50-\mathrm{eV})$ ions detected at the induced magnetosphere boundary near the terminator might instead be related to the plasma clouds observed at the ionopause by the Pioneer Venus orbiter ${ }^{10}$. All of the atmospheric ions moving downstream are accelerated and their original morphological features disappear. However, for escape rate calculations their exact origins are not important as soon as the main escape region has been covered. The orbital scan through the induced magnetosphere does indeed show that it is inside the wake that we observe the highest fluxes of ions (Fig. 1e-g).

The ion escape considered here occurs through two main regions: the plasma sheet and a boundary layer at the induced magnetosphere boundary. These areas are labelled PS and BL in Fig. 3. As expected, the alignment of the plasma sheet plane and the ion energy are controlled by the electric field. Ions of higher energies are in the $+E$ hemisphere and ions of lower energies in the $-E$ hemisphere. All three species show similar spatial distributions, confirming that both $\mathrm{H}^{+}$and $\mathrm{He}^{+}$are indeed of planetary rather than solar-wind origin.

The escape rates for each species, namely $Q\left(\mathrm{O}^{+}\right), Q\left(\mathrm{He}^{+}\right)$and $Q\left(\mathrm{H}^{+}\right)$, are obtained by integrating the flux over $Y_{\mathrm{Vse}}$ and $Z_{\mathrm{Vse}}$. Although the spatial coverage is still not sufficient to obtain the total average escape rate, one can obtain reliable flux ratios. These are $Q\left(\mathrm{H}^{+}\right) / Q\left(\mathrm{O}^{+}\right)=1.9$ and $Q\left(\mathrm{He}^{+}\right) / Q\left(\mathrm{O}^{+}\right)=0.07$.

The escaping plasma consists primarily of $\mathrm{H}^{+}$and $\mathrm{O}^{+}$ions with some admixture of $\mathrm{He}^{+}$. The measured composition differs significantly from the plasma composition in the ionosphere at an ionopause altitude of $300 \mathrm{~km}$ for solar minimum conditions ${ }^{13}$, where the respective number-density ratios are $n\left(\mathrm{H}^{+}\right) / n\left(\mathrm{O}^{+}\right)=0.1$ and $n\left(\mathrm{He}^{+}\right) / n\left(\mathrm{O}^{+}\right)=4 \times 10^{-3}$. The enrichment of the escaping plasma in light species may result from two causes: first, an ion pick-up process operating primarily at higher altitudes, $600-700 \mathrm{~km}$, in which the densities of light species are increased with respect to heavier ions $^{14}$; and second, acceleration by the polarization electric field increasing the flux of lighter ions with respect to heavier because lighter ions gain higher velocities for the same energy.

The ratio of the escape rates of hydrogen and oxygen is a critical parameter in understanding both the dryness and the oxidation state of the venusian atmosphere ${ }^{15}$. Because the main source of hydrogen in the upper atmosphere of Venus is water vapour ${ }^{16}$, two escaping hydrogen atoms would leave one oxygen atom in the atmosphere. Therefore, if the ratio of the escape rate of hydrogen to that of oxygen is greater than the stoichiometric ratio 2:1, there must be other sinks of oxygen, such as surface oxidation.

The measured ratios concern only ions and do not include the escape of the respective atoms. There are two processes that could remove neutral hydrogen and oxygen from the atmosphere to space: loss due to exogenic photochemical and charge-exchange reactions, and atmospheric sputtering. Photochemical and charge-exchange reactions result in a loss of hydrogen at a rate of $50 \%$ of the $\mathrm{H}^{+} \operatorname{loss}^{14}$. Atmospheric sputtering results in an oxygen loss at a rate of $25 \%$ of the $\mathrm{O}^{+} \operatorname{loss}^{14}$. Scaling the measured ratio $\mathrm{Q}\left(\mathrm{H}^{+}\right) / \mathrm{Q}\left(\mathrm{O}^{+}\right)=1.9$ to account for the neutral losses, we arrive at a ratio for the total (neutral atoms plus ions) loss of 2.2:1, very close to the stoichiometric value
2:1. Some excess $\mathrm{H}^{+}$is due to solar-wind protons, which may penetrate through the induced magnetosphere boundary in small amounts and contribute to the measured $\mathrm{H}^{+}$flux in the boundary layer. That the present escape of hydrogen and oxygen through the wake takes place in the stoichiometric ratio of water implies that the atmosphere of Venus did not change its oxidation state after steadystate conditions had been reached, in contrast with that of Mars ${ }^{17}$. This result is thus consistent with the suggestion from atmospheric and ionospheric observations on the Pioneer Venus orbiter ${ }^{15}$ that Venus loses the constituents of water in a ratio that maintains the system's oxidation state.
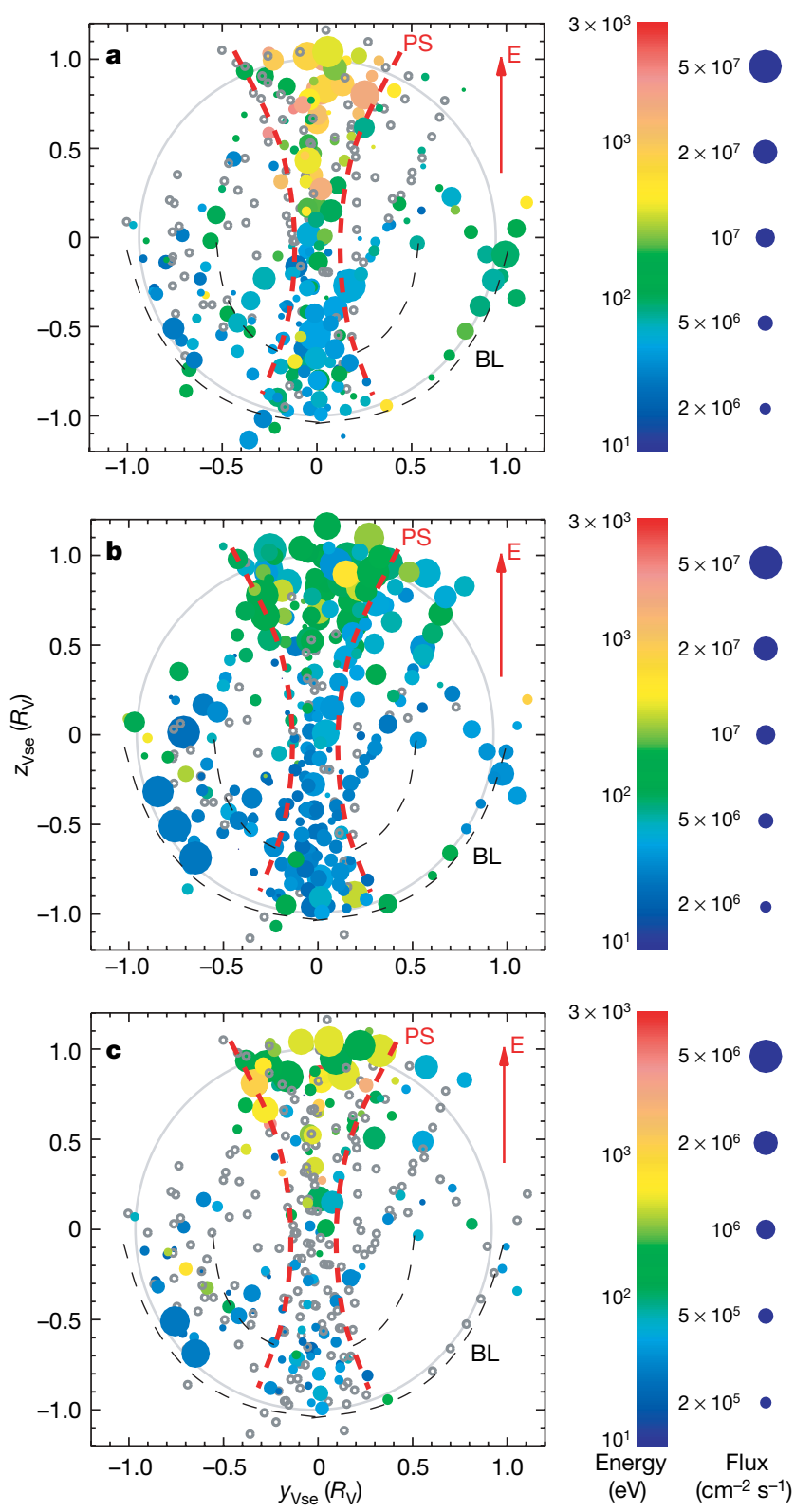

Figure $\mathbf{3}$ | Spatial distribution of the escaping plasma. The measured $\mathrm{O}^{+}$ (a), $\mathrm{H}^{+}$(b) and $\mathrm{He}^{+}$(c) flux distributions in the tail region from 33 orbits were integrated over $X_{\mathrm{Vse}}[-0.5,-3.0]$ and are shown in a $Y_{\mathrm{Vse}}-Z_{\mathrm{Vse}}$ plane across the tail. The geometrical eclipse of Venus is shown by the thin grey circle. To ensure that no solar-wind protons affect the mass composition measurements inside the IMB, we restrict the area of the analysis to $R<1.2 R_{\mathrm{V}}$. Blank circles show measurements with zero flux. The flux scale for $\mathrm{He}^{+}$is tenfold lower than those for $\mathrm{O}^{+}$and $\mathrm{H}^{+}$. The plasma sheet region is identified by red dashed lines and labelled PS, the boundary layer at the IMB is identified by black dashed lines and labelled BL, and the direction of the convection electric field is labelled $\mathrm{E}$. 
The absolute escape rates, including the implications for long-term water evolution, will be determined later. However, the initial analysis gives the reliable lower limit of $10^{25} \mathrm{~s}^{-1}$ for the escape of $\mathrm{H}^{+}$through the plasma wake. Measurements with the Pioneer Venus orbiter's ion and neutral mass spectrometer ${ }^{18}$ and models of the observed tail magnetic and plasma structure ${ }^{19}$ have inferred that there may be copious escape in the wake region. Evidence for hydrogen loss through the pick-up process outside the induced magnetosphere is also seen on Venus Express ( $M$. Delva, personal communication) but the estimated rate of $2 \times 10^{24}$ protons s ${ }^{-1}$ is much lower than that through the wake. Venus therefore loses its water through the plasma wake.

The observed high relative abundance of $\mathrm{He}^{+}$in the escaping plasma from Venus is surprising. At Mars $\mathrm{He}^{+}$is not detected in amounts comparable to the $\mathrm{O}^{+}$flux ${ }^{20}$. The calculated helium ionization rates at Mars $^{21}$ through two channels, photoionization and electron impact ionization, scaled to the total area of Venus, give only an increase in the escaping rate at Venus of a factor of 3 with respect to Mars. The higher abundance of $\mathrm{He}^{+}$may be due to the more effective acceleration caused by the polarization field ${ }^{11}$. The higher pressure gradients associated with the stronger gravitational field on Venus results in a higher polarization field that operates over larger distances at Venus than at Mars. Thus, whereas the stronger gravitational field of Venus reduces the Jeans escape of the heavier main constituents of the atmosphere, almost paradoxically it facilitates the loss of the lighter species.

The main result of these observations, however, is the establishment of potentially important ion escape from present-day Venus through the plasma wake region. Further observations in this region as the solar cycle progresses will establish the variability of these escape rates and will improve our ability to infer the history of Venus's water.

\section{Received 26 June; accepted 29 October 2007.}

1. Donahue, T. M., Grinspoon, D. H., Hartle, R. R. \& Hodges, R. R. Jr. in Venus II: Geology, Geophysics, Atmosphere, and Solar Wind Environment (eds Bougher, W. W., Hunten, D. M. \& Phillips, R. J.) 385-414 (Univ. of Arizona Press, Tucson, AZ, 1997).

2. Nagy, A. F., Cravens, T. E., Lee, J. H. \& Stewart, A. I. P. Hot oxygen atoms in the upper atmosphere of Venus. Geophys. Res. Lett. 8, 629-632 (1981).

3. Mihalov, J. D. \& Brace, A. The distant interplanetary wake of Venus: Plasma observations from Pioneer Venus. J. Geophys. Res. 87, 9045-9053 (1982).

4. Intrilligator, D. S., Wolf, J. H. \& Michalov, J. D. The Pioneer Venus orbiter plasma analyzer experiment. IEEE Trans. Geosci. Remote Sens. GE-18, 39-43 (1980).

5. Kasprzak, W. T., Niemann, H. B., Hedin, A. E., Bougher, S. W. \& Hunten, D. M. Neutral composition measurements by the Pioneer Venus neutral mass spectrometer during re-entry. Geophys. Res. Lett. 20, 2747-2750 (1993).

6. Intriligator, D. S. Results of the first statistical studies of Pioneer Venus plasma observations in the distant Venus tail: Evidence for a hemispheric asymmetry in the pickup of ionospheric ions. Geophys. Res. Lett. 16, 167-170 (1989).
7. Barabash, S. et al. The Analyser of Space Plasmas and Energetic Atoms (ASPERA4) for the Venus Express mission. Planet. Space Sci. 55, 1772-1792 (2007).

8. Zhang, T. L. et al. Magnetic field investigation of the Venus plasma environment: expected new results. Planet. Space Sci. 54, 1336-1343 (2006)

9. Zhang, T. L. et al. Little or no solar wind enters Venus' atmosphere at solar minimum. Nature doi:10.1038/nature06026 (this issue).

10. Brace, L. H., Theis, R. F. \& Hoegy, W. R. Plasma clouds above the ionopause of Venus and their implications. Planet. Space Sci. 30, 29-37 (1982).

11. Hartle, R. E. \& Grebowsky, J. M. Planetary loss from light ion escape on Venus. Adv. Space Res. 15, 117-122 (1995).

12. Luhmann, J. G., Ledvina, S. A., Lyon, J. G. \& Russell, C. T. Venus $\mathrm{O}^{+}$pickup ions: Collected PVO results and expectations for Venus Express. Planet. Space Sci. 54, 1457-1471 (2006)

13. Fox, J. L. Advances in the aeronomy of Venus and Mars. Adv. Space Res. 33, 132-139 (2004).

14. Lammer, H. et al. Loss of hydrogen and oxygen from the upper atmosphere of Venus. Planet. Space Sci. 54, 1445-1456 (2006).

15. McElroy, M. B., Prather, M. J. \& Rodriguez, J. M. Loss of oxygen from Venus. Geophys. Res. Lett. 9, 649-651 (1982).

16. von Zahn, U., Kumar, S., Niemann, H. \& Prinn, R. in Venus (eds Hunten, D. M., Colin, L., Donahue, T. M. \& Moroz, V. I.) 299-430 (Univ. of Arizona Press, Tucson, AZ, 1983).

17. Lammer, H. et al. Loss of water from Mars: Implications for the oxidation of the soil. Icarus 165, 9-25 (2003).

18. Luhmann, J. G., Kasprzak, W. T. \& Russell, C. T. Space weather at Venus and its potential consequences for atmosphere evolution. J. Geophys Res. 112, E04s10, doi:10.1029/2006JE002820 (2007).

19. McComas, D. J., Spence, H. E., Russell, C. T. \& Saunders, M. A. The average magnetic field draping and consistent plasma properties of the Venus magnetotail. J. Geophys. Res. 91, 7939-7953 (1986).

20. Barabash, S., Fedorov, A., Lundin, R. \& Sauvaud, J.-A. Martian atmospheric erosion rates. Science 315, 501-503 (2007).

21. Krasnopolsky, V. A. \& Gladstone, G. R. Helium on Mars and Venus: EUVE observations and modeling. Icarus 176, 395-407 (2005).

22. Zhang, T. L., Luhmann, J. G. \& Russell, C. T. The solar cycle dependence of the location and shape of the Venus bow shock. J. Geophys. Res. 95, 14961-14967 (1990).

23. Zhang, T. L., Luhmann, J. G. \& Russell, C. T. The magnetic barrier at Venus. J. Geophys. Res. 96, 11145-11153 (1991).

Supplementary Information is linked to the online version of the paper at www.nature.com/nature.

Acknowledgements We thank the European Space Agency for providing the Venus Express opportunity, and national space agencies and organizations for supporting the investigators who contributed to the success of the Venus Express plasma package.

Author Contributions S.B. is the principal investigator of the Venus Express plasma package, Analyser of Space Plasmas and Energetic Atoms (ASPERA)-4. J.A.S. is the co-principal investigator. A.F. is the leading co-investigator of the ion mass analyser of ASPERA-4. A.C. is the leading co-investigator of the electron spectrometer of ASPERA-4. T.L.Z. is the principal investigator of the magnetometer. The remaining authors are co-investigators on either of the plasma analyser or the magnetometer investigations.

Author Information Reprints and permissions information is available at www.nature.com/reprints. Correspondence and requests for materials should be addressed to S.B. (stas@irf.se). 